\title{
Desarrollo y aplicación del software DISEVAP_edu como apoyo al aprendizaje del diseño y análisis de procesos de evaporación de múltiples efectos
}

\author{
José M. Gozálvez Zafrilla ${ }^{a}$, Asunción Santafé Moros ${ }^{a}$, David Catalán Martínez ${ }^{b}$, Fidel \\ Toldrá Reig ${ }^{\mathrm{b}}$, Manuel Cesar Martí Calatayud ${ }^{\mathrm{a}}$ \\ ${ }^{a}$ Equipo de Innovación y Calidad Educativa ASEI. Departamento de Ingeniería Química y Nuclear. \\ Instituto de Seguridad Industrial y Medioambiental. E.T.S. Ingenieros Industriales de la Universitat \\ Politècnica de Valencia, jmgz@iqn.upv.es, assanmo@iqn.upv.es 'Instituto de Tecnología Química- \\ CSIC. Universitat Politècnica de Valencia, dacamar3@itq.upv.es, fitolrei@itq.upv.es.
}

\begin{abstract}
Evaporation is an important process operation taught in the subject "Extension on Separation Operations" of the Master in Chemical Engineering of the UPV. Main teaching objectives concerning to this operation are to deepen in the design and operation of the multi-effect evaporation systems. To cover successfully these objectives and to help in the development of transversal competences, the ASEI team has developed the DISEVAP software that allows you to evaluate the evaporation configurations faster and to study the effect of the most influential factors on the process performance. In the present article, the organizational changes within the subject that have been used in a reflection on how to improve the use of software to develop the skills related to reasoning are detailed. Likewise, the methodology for obtaining information on the level of competence acquired by the student is detailed, which has allowed comparing teaching results obtained using the program with those of previous years. The results indicate that the methodology based on the use of the program has achieved an improvement in teaching. However, it is still necessary to make certain readjustments to develop its maximum potential in coming years.
\end{abstract}

Keywords: evaporation, Chemical Engineering, Desalination, Separation operations, competences, software, methodology, evaluation.

\footnotetext{
Resumen

La evaporación es una importante operación de proceso enseñada en la asignatura "Ampliación de Operaciones de Separación" del Máster Universitario de Ingeniería Química de la UPV. Los principales objetivos docentes de Máster concernientes a esta operación son profundizar en el diseño y funcionamiento de sistemas de múltiples efectos. Para poder cubrir estos objetivos docentes satisfactoriamente y ayudar en el desarrollo de competencias transversales, el equipo ASEI ha desarrollado el software DISEVAP, el cual permite evaluar con rapidez diferentes configuraciones de evaporación y estudiar el efecto de los factores más influyentes sobre las
} 
prestaciones del proceso. En el presente artículo se detallan los cambios organizativos dentro de la asignatura que han sido necesarios haciendo una reflexión sobre cuál es la mejor forma de aprovechar el software para desarrollar competencias relacionadas con el razonamiento. Asimismo, se detalla la metodología de obtención de información sobre el nivel de competencia adquirido por el alumno, la cual ha permitido comparar resultados docentes obtenidos utilizando el programa con los de años previos. Los resultados indican que la metodología basada en el uso del programa ha logrado una mejora de la docencia, si bien, son necesarios ciertos reajustes para desarrollar su máximo potencial en años venideros.

Palabras clave: evaporación, Ingeniería Química, desalación., operaciones de separación, competencias, software, metodología, evaluación.

\section{Introducción}

\subsection{La operación de evaporación, su cálculo y diseño}

La evaporación es una operación importante en Ingeniería Química y de Procesos. Consiste en el paso a vapor de parte del líquido de una disolución mediante suministro de calor a una presión adecuada con el objetivo de concentrarla en los componentes no volátiles o bien de recuperar el líquido (Santafé-Moros et al., 2013). La evaporación es empleada en la industria química, alimentaria, minera, papelera y farmacéutica para obtener líquidos concentrados (Brian and Hackett, 2018). Puede realizarse en un único evaporador (efecto) o mediante una serie de efectos que pueden disponerse en diferentes configuraciones según la secuencia de calentamiento y de intercambio de corrientes líquidas entre éstos.

Una buena comprensión de la operación requiere siempre repasar conceptos termodinámicos y físico-químicos previamente a la explicación de los cálculos (de Busto, 2008). Para la operación de un solo efecto es necesario saber plantear los balances de materia, componente y energía, así como dominar la transmisión de calor y la resolución de sistemas de ecuaciones algebraicas. El cálculo de sistemas de múltiples efectos es bastante más complicado que el de un efecto aislado al añadirse la complejidad de la interacción entre subsistemas. En distintas operaciones de separación (absorción, destilación, extracción, ...) existen algoritmos para determinar la configuración de etapas óptima. En cambio, para la evaporación de múltiples efectos no es posible determinarla a priori, lo que obliga a realizar un barrido de cálculos de configuraciones para realizar un diseño óptimo.

Por otra parte, el cálculo de cada una de las configuraciones requiere la determinación de las necesidades de vapor de calefacción, área de intercambio y presiones de operación de los efectos a través de un algoritmo de tipo iterativo. Esto implica la necesidad de dominar diversos conocimientos:

- conceptos físico-químicos, termodinámicos y de transmisión de calor

- conocimiento de los equipos

(c) BY-NC-ND 2019, Universitat Politècnica de València 
- diseño de la configuración y variables operativas de los procesos

- programación de funciones de propiedades y cálculos iterativos

- economía y optimización de los procesos

Finalmente, cabe añadir que existen dos modalidades diferentes de evaporación que emplean múltiples efectos: la evaporación multiefecto en evaporadores de tubos y la evaporación súbita multietapa (también conocida como flash) difiriendo su cálculo sustancialmente. Normalmente los cálculos de la segunda modalidad no se explican, incluso en asignaturas de desalación donde resulta más relevante.

\subsection{Situación actual en la enseñanza en la ETSII de la UPV}

Los contenidos docentes relacionados con esta operación se imparten principalmente en asignaturas que recaen bajo el nombre de "operaciones de separación" u "operaciones básicas o unitarias" de las titulaciones de Ingeniería Química, pero también en otras relacionadas con desalación, procesos industriales o agroalimentarios de otras titulaciones. En el caso concreto de la E.T.S. de Ingenieros Industriales de la UPV se imparten en el Grado de Ingeniería Química (GIQ) y en el Máster Universitario en Ingeniería Química (MUIQ).

En el GIQ, se explican los fundamentos físicos de la operación, los equipos más usuales, y se enseña el cálculo de la operación de un solo efecto de evaporación utilizando el entorno de cálculo matemático Mathcad, el cual permite presentar con una notación muy cercana a la matemática convencional, los diferentes cálculos (Santafé-Moros et al., 2015).

En el nivel de Máster se profundiza en los fundamentos físicos, y lo que es más importante se pasa a trabajar con sistemas de múltiples efectos introduciéndose dos tipos de sistemas: sistemas de evaporación por destilación multiefecto y sistemas de evaporación súbita multiefecto. En ambos tipos de sistema las condiciones de cada efecto influyen en las de los adyacentes lo que implica que el cálculo sea iterativo. Esta circunstancia, unida a que los algoritmos de resolución son de tipo iterativo, hace que sea necesario un elevado tiempo para completar los procedimientos de cálculo, lo cual limita el tiempo disponible para el aprendizaje conceptual del diseño crítico y de los factores que determinan el comportamiento del proceso. Hasta ahora esto se ha solventado parcialmente gracias a la utilización estructurada del software Mathcad para realizar cálculos de diseño enfocados al dimensionamiento del proceso para una alimentación, configuración y salida de producto previamente definidas (Gozálvez-Zafrilla et al., 2015). No obstante, el nivel de competencias deseado a nivel de máster es que el alumno sea capaz de analizar críticamente los resultados y que los diseños se hagan no sólo desde el punto de vista de cálculo, sino que se tenga capacidad de considerar múltiples criterios entre ellos el económico. Estos últimos objetivos no se llegaban a cubrir hasta ahora satisfactoriamente debido a limitaciones de tiempo en la asignatura.

Otra deficiencia importante es que no se llegaban a realizar cálculos de simulación para estudiar la respuesta del sistema diseñado frente a cambios en la alimentación de entrada o 
en las condiciones operativas, perdiéndose la capacidad que presenta la simulación como herramienta docente para facilitar la comprensión del funcionamiento del proceso.

\section{Objetivos}

La innovación docente se diseñó principalmente para la parte práctica de la asignatura "Ampliación de Operaciones de Separación" del Máster de Ingeniero Químico de la Universitat Politècnica de Valencia. Para las sesiones correspondientes al tema de evaporación se planteó como objetivo general alcanzar de forma efectiva las siguientes competencias de nivel de máster:

- La capacidad de obtener diseños óptimos de sistemas de evaporación desde un punto de vista económico

- La comprensión de los efectos de las variables operativas y de la configuración

- La capacidad de reflexionar críticamente sobre resultados obtenidos".

Este objetivo se planteó bajo las restricciones de que debía continuar desarrollándose dentro de la sesión práctica y que además no se debía menoscabar la enseñanza de los conceptos y metodologías de cálculo explicadas hasta ahora.

\section{Desarrollo de la innovación}

El objetivo planteado junto con sus restricciones requiere reestructurar la metodología de la clase y aumentar la eficacia de los cálculos realizados de forma que se consiga tiempo para evaluar un número de suficiente de configuraciones y reflexionar sobre los resultados. Tras una fase previa de análisis se determinó la necesidad de realizar un software de apoyo y los necesarios cambios estructurales en la sesión práctica y la metodología de evaluación.

A continuación, se exponen de forma más detallada las acciones llevadas a cabo:

\subsection{Análisis previo}

En primer lugar, se analizaron las sesiones teóricas y se valoró si era necesario realizar cambios en éstas. Se llegó a la conclusión de que no era conveniente realizar modificaciones relevantes, manteniéndose la enseñanza de los conceptos y de los cálculos de evaporación multiefecto como estaba, salvo aquellas destinadas a describir de forma breve el planteamiento actual de las sesiones prácticas y solicitar el trabajo previo necesario.

En el análisis de las sesiones prácticas se consideró conveniente mantener el trabajo en parejas, pues toda la experiencia previa indicaba que resulta más efectiva que el trabajo individual en prácticas de laboratorio informático de este tipo en las que se deben realizar cálculos y discutir sobre los resultados.

Se identificaron tareas excesivamente repetitivas en las que se consumía una gran parte del tiempo de prácticas. En la metodología previa se completaba en Mathcad una plantilla con el 
procedimiento de cálculo explicado en las sesiones teóricas para una configuración de tres efectos. Este procedimiento se realizaba en las sesiones prácticas con asistencia del profesor. Posteriormente le correspondía a cada pareja de alumnos adaptar el mismo fichero a diferentes configuraciones, identificando los puntos del cálculo a modificar y trasladando los resultados obtenidos para las distintas configuraciones a una hoja Excel para su análisis. Las gráficas obtenidas debían subirse a PoliformaT y el alumno debía acabar concluyendo cual era la configuración más conveniente entre las calculadas. La labor de adaptación de ficheros de cálculo para cada configuración consumía casi el $75 \%$ del tiempo de prácticas, siendo percibido adicionalmente como una tarea aburrida por parte de los alumnos. Aunque esta labor de adaptación conduce a una comprensión adecuada del proceso de cálculo, se decidió reducirla a un mínimo para conseguir el tiempo necesario para realizar otras actividades.

A continuación, se analizó en base a los resultados obtenidos en exámenes y prácticas anteriores cuales eran los puntos débiles susceptibles de mejorarse con las nuevas actividades, y se detectaron los siguientes en un número bastante significativo de alumnos:

- Existencia de dudas conceptuales graves sobre los efectos que causa la modificación de un parámetro operativo o de la alimentación del proceso.

- Incapacidad de plantear una estrategia para llegar a una solución óptima en un conjunto de discreto de posibles combinaciones.

- Dudas a la hora de plantear una función objetivo adecuada para llegar a una solución óptima con desconocimiento de algunos de los datos necesarios (a lo que se sumaría la dificultad en saber encontrarlos, p. ej. en Internet)

La primera problemática expuesta es consecuencia de que algunos efectos no son evidentes, y se pensó que podría ser de gran ayuda disponer de demostraciones rápidas con el programa de cálculo para pasar a reflexionar posteriormente. El problema para hacerlo era que los algoritmos de cálculo de evaporación empleados estaban enfocados al diseño y con ellos es difícil lograr obtener el efecto de modificar variables operativas si no se realiza un procedimiento iterativo. Se concluyó que era necesario introducir una herramienta que permitiese la simulación directa de estos efectos.

La segunda problemática puede solventarse planteando un problema de este tipo en la práctica y explicando brevemente una estrategia de búsqueda de soluciones óptimas. No obstante, para ello es necesario un sistema rápido en la obtención de soluciones y el sistema utilizado hasta el momento no lo cumplía, pues aparte del elevado tiempo necesario, los alumnos se perdían entre el cálculo de las configuraciones y la propia estrategia de obtención de la configuración óptima. En este caso es necesario un código de cálculo que permita la realización rápida de cálculos de tipo diseño.

La tercera problemática se trabaja conjuntamente con la segunda pues la correcta definición de una función objetivo es intrínseca a cualquier problema de optimización, pero es necesario disponer de un tiempo suficiente si se desea dejar al alumno que concrete más el problema y busque los datos, extremo por otra parte deseable como competencia de nivel de máster. 


\subsection{Desarrollo y descripción del software DISEVAP_edu}

Como herramienta de apoyo necesaria se pensó en un primer momento en la utilización de un simulador de procesos convencional ya que algunos son una herramienta excelente para demostraciones de la simulación en docencia. No obstante, aunque técnicamente es posible realizar el cálculo de un sistema de evaporadores en este tipo de software se descartó por las circunstancias que a continuación se plantean. En primer lugar, el cálculo de tipo diseño es poco directo, debiéndose tantear algunos parámetros. En segundo lugar, resultan poco adecuados cuando se ha de calcular un número importante de configuraciones de proceso, pues cada vez debe realizarse gráficamente un diagrama de flujo del proceso en el propio entorno. En tercer lugar, en el caso de sistemas de evaporación la implementación de condiciones de funcionamiento requiere la implementación de condiciones que en el sistema industrial realizan los controladores. Todo ello nos llevaría también a una tarea repetitiva con los problemas ya comentados.

Por tanto, se decidió emplear un código de simulación para su utilización docente que reuniese tanto la capacidad de diseñar como de simular sistemas de evaporación. Este código es una adaptación docente del código DISEVAP realizado por uno de los miembros del grupo ASEI. La versión estudiante es un ejecutable realizado con Matlab Compiler. La Fig. 1 muestra la pantalla de inicio en la que se ofrece la posibilidades de buscar un fichero de datos o bien abrir dos casos ejemplo: uno de un sistema de evaporación multiefecto de tubos y otro de un sistema de evaporación súbita multietapa. Los casos ejemplo se pensaron para la versión estudiante por representar dos casos importantes en Ingeniería Química (concentración de sosa para el caso multiefecto y desalación para el caso de evaporación súbita) ampliamente documentados. Como se puede ver, la presentación de la versión actual 1.1 resulta poco atractiva por el fondo negro, estando previsto utilizar las GUI de Matlab en un futuro para llegar a un programa de más fácil interacción. No obstante, por el momento se ha logrado una interacción aceptable gracias a la capacidad de Matlab de interacción con Excel o editores de ficheros CSV (ficheros de valores separados por comas).

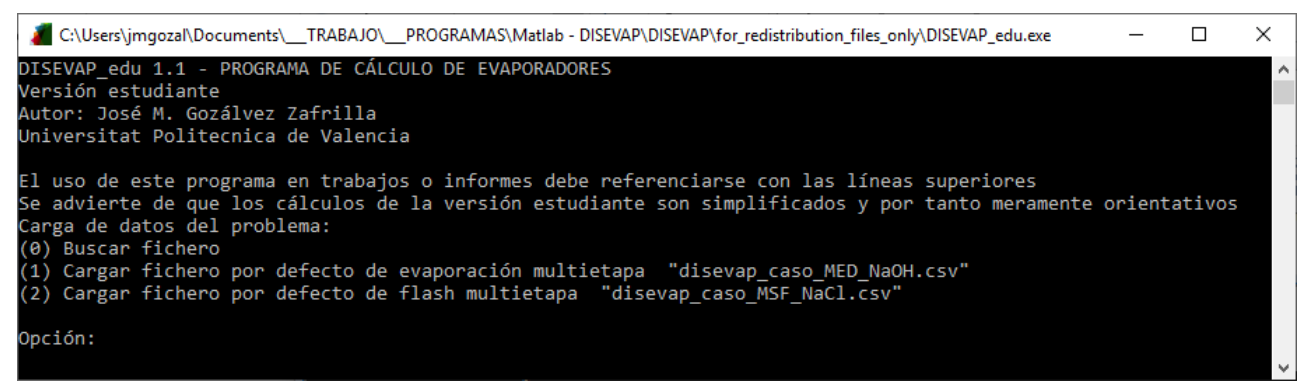

Fig. 1 Pantalla de inicio de la versión educacional de DISEVAP_edu

Todos los datos necesarios para la resolución de un problema se estructuran en un fichero principal (Fig. 2) y cinco ficheros de datos adicionales de tipo CSV. El fichero principal contiene los datos básicos de componentes, entrada, tipo de intercambiador, configuración, 
condiciones operativas. Como se puede ver es posible trabajar con diferente tipo de unidades para cada variable acompañando el valor numérico por una expresión de unidades en la celda contigua, lo cual siempre se agradece por el usuario. El fichero principal contiene también los nombres de ficheros de propiedades necesarios (intercambio de calor, elevación del punto de ebullición, así como entalpía, viscosidad y densidad de disoluciones). Estos ficheros de datos utilizados son fácilmente adaptables a otras disoluciones diferentes de las utilizadas como casos ejemplo si se dispone de datos empíricos. Los datos y correlaciones necesarios correspondientes al agua líquida o vapor están incorporados dentro del propio software.

\begin{tabular}{|c|c|c|c|}
\hline 4 & A & B & $\mathrm{C}$ \\
\hline 1 & Soluto & $\mathrm{NaOH}$ & \\
\hline 2 & F, Flujo de entrada y unidades & 10000 & $\mathrm{~kg} / \mathrm{h}$ \\
\hline 3 & XF, Fracción de componente en la entrada & 0.1 & \\
\hline 4 & TF, Temperatura de entrada y unidades & 37.8 & $\stackrel{\circ}{C}$ \\
\hline 5 & xLs, Fracción objetivo a la salida: & 0.3 & \\
\hline 6 & Tipo (MED|MSF) & MED & \\
\hline 7 & Configuración & {$\left[\begin{array}{lll}1 & 2 & 3\end{array}\right]$} & \\
\hline 8 & Pesos de área & [1 1111$]$ & \\
\hline 9 & pS, Presión absoluta del vapor vivo y unidades & 132.39 & $\mathrm{kPa}$ \\
\hline 10 & dTS, Sobrecalentamiento del vapor vivo y unidades & 0 & $\stackrel{\mathrm{C}}{ }$ \\
\hline 11 & pcond, Presión absoluta del condensador y unidades & 6.666 & $\mathrm{kPa}$ \\
\hline 12 & Unid. potencia para los resultados: & $\mathrm{MJ} / \mathrm{h}$ & \\
\hline 13 & Fichero de datos de intercambio de calor & \multicolumn{2}{|c|}{ disevap_intercambio } \\
\hline 14 & Fichero de datos de elevación del punto de ebullición & \multicolumn{2}{|c|}{ disevap_NaOH_DTB } \\
\hline 15 & Fichero de datos de entalpía & \multicolumn{2}{|c|}{ disevap_NaOH_h } \\
\hline 16 & Fichero de datos de viscosidad de disoluciones & \multicolumn{2}{|c|}{ disevap_NaOH_visco } \\
\hline 17 & Fichero de datos de densidad de disoluciones & \multicolumn{2}{|c|}{ disevap_NaOH_dens } \\
\hline
\end{tabular}

Fig. 2 Ejemplo de fichero principal de DISEVAP_edu

Como fichero de principal puede escogerse uno de los casos predeterminados o cualquier otro fichero de caso si se selecciona la opción de escogerlo desde un explorador de archivos. Una vez cargado los datos se pasa inmediatamente al cálculo del problema (Fig. 3). El cálculo proporciona las condiciones físicas de los fluidos en los evaporadores y las necesidades de área y vapor vivo. Al final del cálculo se ofrecen dos opciones. Con la primera es posible modificar la configuración cambiando la secuencia de líquidos, siendo esto posible tanto para el mismo número de efectos como para otro cualquiera, bastando con añadir una sucesión factible de efectos y sin necesidad de tener que modificar el CSV. Con la segunda es posible repetir el cálculo sin imponer que los efectos sean iguales indicando la relación de área entre efectos. En nuestro conocimiento no hemos constatado esta capacidad en ningún otro código.

Así se puede seguir calculando hasta encontrar una solución que logre una optimización adecuadad del problema de acuerdo con la función objetivo definida. Seleccionada la opción de cierre del modo diseño se pasa a modo simulación donde es posible realizar un análisis del efecto de las variables principales sobre el diseño efectuado. Como ejemplo, en la Fig. 4 se muestra la realización de un cambio en el flujo de alimentación y los resultados del posterior recálculo. Posteriormente, se puede continuar analizando el efecto de otra variable 
Desarrollo y aplicación del software DISEVAP como apoyo a la enseñanza de los sistemas de evaporación de múltiples efectos

devolviendo la variable flujo a su valor original y modificando la nueva variable sin necesidad de modificar los ficheros CSV cada vez.

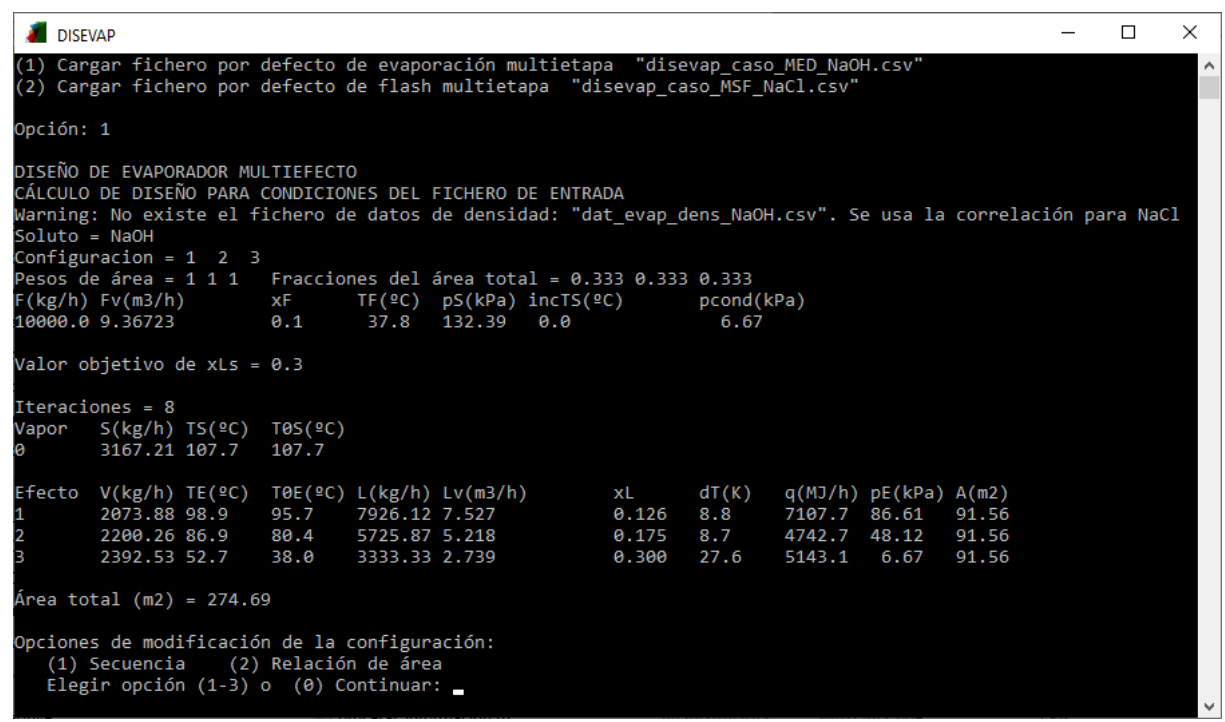

Fig. 3 Ejemplo de diseño realizado por DISEVAP_edu

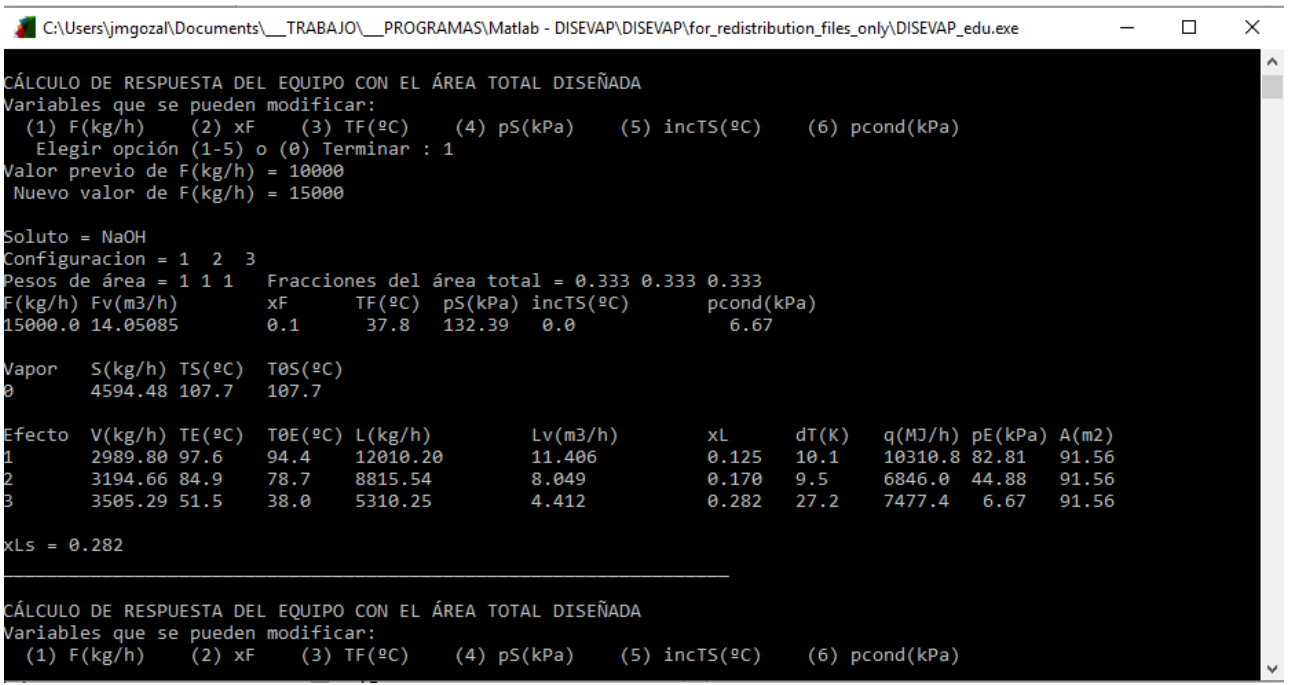

Fig. 4 Efecto de estudio del efecto de una variable operativa calculado por DISEVAP_edu

Todos los cálculos realizados quedan almacenados en un fichero log para un posterior análisis. La estructura de esta fichero de texto es tal que permite copiar directamente zonas a casillas de Excel para su posterior procesamiento. 


\subsection{Adaptación de la estructura y metodología de las sesiones prácticas}

Se consideró conveniente mantener sin modificaciones la parte inicial de la práctica destinada al aprendizaje práctico del cálculo. En ésta se completa una plantilla en Mathcad para calcular un sistema de efectos en configuración 1-2-3. Se mantiene el uso de Mathcad por coherencia con el resto de la asignatura donde se exponen métodos de cálculo de procesos muy diferentes y donde resulta muy adecuado utilizar la posibilidad que Mathcad ofrece de una notación representación matemática casi igual a la convencional junto con la integración de gráficos y texto de ayuda. Esta claridad, que no se puede lograr con lenguajes como Matlab o Python, queda patente en la Fig. 5 donde se muestra la resolución de un sistema de ecuaciones que aparece en el proceso. El uso de zonas favorece además la estructuración del problema (Santafé-Moros et al., 2015).

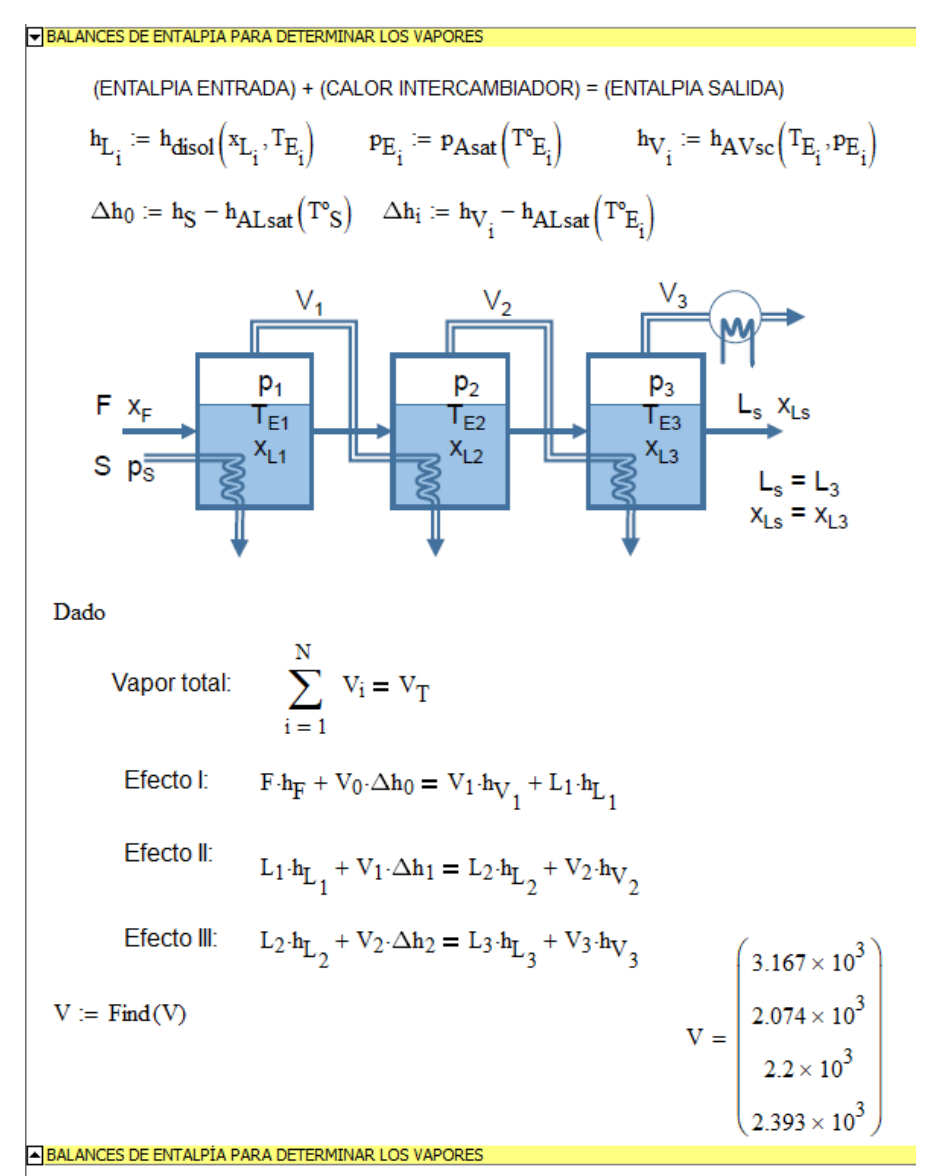

Fig. 5 Zona de cálculo de Mathcad

En cambio, se decidió reducir sustancialmente la labor de cálculo de otras configuraciones mediante la modificación del fichero problema, procediéndose en su lugar a realizar las siguientes actividades: 
1. Realización de una única modificación de fichero para realizar el cálculo de una configuración y su comparación con los resultados calculados con DISEVAP en modo diseño.

2. El cálculo posterior de un número elevado de configuraciones empleando DISEVAP y la evaluación del coste asociado a cada configuración.

3. Estudio de los efectos de las variables operativas y la alimentación en la configuración diseñada.

En la primera actividad el profesor reflexiona brevemente con los alumnos sobre los puntos a cambiar en el fichero para adaptar el proceso señalándolos en el proyector. Los alumnos tras adaptarlo debían calcular el nuevo diseño con DISEVAP y comparar los resultados (el resultado no es plenamente coincidente por ser los métodos del simulador más rigurosos). Uno de los dos alumnos debe subir el fichero modificado y la discusión de diferencias a preguntas de un examen abierto en la sesión de prácticas en la plataforma PoliformaT. Se les informa, al igual que se hacía en años anteriores, que era un conocimiento exigible para el examen y que debían practicar.

Para la segunda actividad, en la clase de teoría los alumnos han debido buscar datos de costes de vapor o de unidades de evaporación. Esta labor de búsqueda previa está, por otra parte, asociada a la evaluación de una de las actividades realizadas dentro de la competencia transversal "Aprendizaje permanente" de la asignatura. El profesor comienza con una reflexión junto con los alumnos sobre los factores más importantes en el coste y se deja un tiempo para que el alumno busque datos adicionales y defina la función objetivo. Se dan algunas indicaciones para definir una estrategia para llegar hasta la configuración óptima. Los resultados obtenidos deben subirse al mismo examen abierto de PoliformaT.

Con el tiempo ganado gracias a la utilización del software es posible plantear una tercera actividad para estudiar los efectos de las variables operativas y la alimentación en una configuración indicada por el profesor. En este caso se puede utilizar el programa DISEVAP_edu en modo simulación.

En la práctica concreta de este curso, a efectos demostrativos se hizo también un cálculo de diseño de un evaporador por evaporación súbita y se comparó con el evaporador de múltiples efectos.

\subsection{Evaluación del alumnado y de la propia acción}

Para la evaluación se utilizó un método similar al utilizado hasta ahora basado en la utilización de un examen abierto en la plataforma PoliformaT durante la propia práctica. Fue necesario cambiar el tipo de preguntas, aumentándose la importancia de las zonas de texto dedicadas a la discusión.

Los resultados de la propia acción se evaluaron pidiendo opiniones particulares a los alumnos y a través de la comparación de los resultados de examen referentes a esta parte con los de años anteriores. 


\section{Resultados}

\subsection{Impacto de la acción en la competencia del alumnado}

Bajo la máxima primum non nocere, la principal preocupación tras la implantación de la nueva metodología fue comprobar que no se había producido un deterioro significativo en el nivel de competencia que se estaba teniendo en la habilidad de calcular distintas configuraciones. En este caso se podía utilizar los resultados de examen de convocatorias previas donde se pone un problema de examen basado en realizar algunas de las modificaciones practicadas. Los resultados uniformizados en base diez de la nota de la cuestión correspondiente quedan reflejados en la Tabla 1. De acuerdo con estos resultados la media ponderada de los cursos anteriores bajo la antigua metodología resultó ser de 7.3 con una desviación estándar de 3.0. Por lo tanto, no se puede afirmar que la habilidad de cálculo en el nuevo curso haya quedado afectada significativamente por los cambios.

Tabla 1. Resultados de examen de problema de cálculo de evaporación (base 10)

\begin{tabular}{cccc}
\hline Convocatoria & Muestra & Media & $\begin{array}{c}\text { Desviación } \\
\text { estándar }\end{array}$ \\
\hline $2018-19 *$ & 53 & 7.7 & 2.5 \\
$2017-18$ & 42 & 8.1 & 3.1 \\
$2016-17$ & 42 & 6.1 & 3.1 \\
$2015-16$ & 25 & 7.6 & 1.6 \\
\hline
\end{tabular}

*Curso en el que se han aplicado los cambios

En cambio, sí que se ha observado una mejora en la asimilación de conceptos relacionados con la respuesta del sistema frente a cambios operativos. Los alumnos obtuvieron una respuesta media con una nota que sobrepasaba en más de un punto a la del curso anterior que fue el único en el que se solicitó una actividad similar (Tabla 2).

Sin embargo, los mejores resultados de aprendizaje se obtuvieron en la actividad enfocada a la obtención de soluciones óptimas de configuración donde era de esperar que tuviera mayor efecto la introducción de la metodología de trabajo con el software desarrollado. La Tabla 3 muestra resultados de evaluación del curso presente y de dos cursos anteriores donde se evaluó de forma estructurada esta actividad. Con la nueva metodología todos los alumnos obtuvieron un número suficiente de configuraciones y dispusieron de tiempo necesario para la reflexión. Las notas bajas en este apartado en la antigua metodología eran sobretodo debidas a que era la última actividad exigida y muchos alumnos ni siquiera llegaban a cumplimentarla. 
Desarrollo y aplicación del software DISEVAP como apoyo a la enseñanza de los sistemas de evaporación de múltiples efectos

Tabla 2. Resultados en la práctica de actividad relacionada con la comprensión de efecto de la evaporación (15\% nota práctica, base 10$)$

\begin{tabular}{ccccc}
\hline Convocatoria & $\begin{array}{c}\text { \%Nota } \\
\text { práctica }\end{array}$ & Muestra & Media & $\begin{array}{c}\text { Desviación } \\
\text { estándar }\end{array}$ \\
\hline $2018-19 *$ & $15 \%$ & 53 & 8.0 & 3.9 \\
$2017-18$ & $10 \%$ & 40 & 6.8 & 5.8 \\
\hline
\end{tabular}

*Curso en el que se han aplicado los cambios

Tabla 3. Resultados en la práctica de actividad de obtención de soluciones óptimas de evaporación (base 10)

\begin{tabular}{ccccc}
\hline Convocatoria & $\begin{array}{c}\text { \%Nota } \\
\text { práctica }\end{array}$ & Muestra & Media & $\begin{array}{c}\text { Desviación } \\
\text { estándar }\end{array}$ \\
\hline $2018-19^{*}$ & $20 \%$ & 53 & 8.9 & 3.0 \\
$2017-18$ & $10 \%$ & 40 & 5.3 & 1.9 \\
$2016-17$ & $15 \%$ & 44 & 5.5 & 2.1 \\
\hline
\end{tabular}

*Curso en el que se han aplicado los cambios

\subsection{Actitudes y opinión del alumnado}

Lamentablemente no se realizó ninguna encuesta durante la asignatura. No obstante sí que se recogen a continuación impresiones del alumnado:

- La forma de trabajo (secuencia de opciones, uso de ficheros CSV no resultó excesivamente dificultosa a los alumnos.

- El fondo negro del programa (consecuencia de no estar implementada una interfaz gráfica GUI) les resultaba poco estético y les dio una primera impresión de que la aplicación era primitiva.

- No hubo protestas por falta de tiempo en la práctica para cumplimentar las tareas, a diferencia de otros años donde eran generalizadas

- No hubo ninguna queja por parecer la práctica monótona (lo cual fue esporádico en años anteriores)

- Los alumnos valoran muy positivamente que la memoria de la práctica siga realizándose online y dentro del tiempo de prácticas.

\section{Conclusiones}

La metodología basada en la utilización del programa DISEVAP_edu en prácticas de evaporación multiefecto del Máster Universitario de Ingeniería Química ha supuesto una 
mejora de los resultados docentes. Por una parte, permite una mejor comprensión del funcionamiento de estos sistemas a través de la capacidad de observar el efecto de parámetros operativos en su modo de simulación. Por otra, permite plantear una estrategia para la obtención de diseños óptimos de este tipo de sistemas. La liberación de tiempo de tareas que el alumno puede realizar por sí solo aparte del tiempo de prácticas ha permitido la introducción de actividades relacionadas con el razonamiento y el pensamiento crítico y competencias transversales.

\section{Referencias}

BRIAN, W., HACKETT, P.E., (2018). "The Essentials of Continuous Evaporation". CEP Magacine (AIChE Publ. https://www.aiche.org/resources/publications/cep/2018/may/essentials-continuousevaporation

DE BUSTO, A.L.R., (2008). "Diseño de una propuesta didáctica y su contribución a la enseñanza y aprendizaje del tema Equilibrio Químico, para alumnos que ingresan en la Universidad. Univ Santiago de Compostela".

GOZÁlVEZ-ZAFRILlA, J.M., SANTAFÉ-MOROS, A., NAVARRO-LABOULAIS, J., (2015). "Adaptación de metodologías docentes para la enseñanza de problemas en aula informática con el objetivo de formar en competencias de nivel de Máster", en: Congreso Nacional de Innovación Educativa y Docencia En Red (IN-RED 2015). Editorial Universitat Politècnica de València, Valencia, pp. $1-15$.

HARB, J.N., JONES, A., ROWLEY, R.L., WILDING, W.V., (1997)." Use of computational tools in engineering education. A case study on the use of Mathcad" Chem. Eng. Educ. 180-187.

SANTAFÉ-MOROS, A., GOZÁLVEZ-ZAFRILLA, J.M., (2015a). "Developing student outcomes in problem sessions of Separation Processes. Comparative study between Bachelor and Master degrees" en International Congress on Education, Innovation and Learning Technologies (ICEILT 2015). ScienceKNOW Conferences C.B., Granada (España), pp. 68-68.

SANTAFÉ-MOROS, A., GOZÁLVEZ-ZAFRILLA, J.M., (2015b). "Aplicación de metodologías para el aprendizaje de problemas de operaciones de separación" en: I Jornadas de Innovación Docente Campus Iberus y IX Jornadas de Innovación Docente e Investigación Educativa de la Universidad de Zaragoza. Universidad de Zaragoza, Zaragoza (España), pp. 36-36.

SANTAFÉ-MOROS, A., GOZÁLVEZ-ZAFRILLA, J.M., LORA-GARCÍA, J., (2013). "Cálculo de operaciones de separación con Mathcad". Editorial Universitat Politècnica de València.

SANTAFÉ-MOROS, A., GOZÁlVEZ-ZAFRILlA, J.M., NAVARRO-LABOULAIS, J., (2015). "Análisis estructural de los problemas orientado al aprendizaje de Operaciones de Separación" en Congreso Nacional de Innovación Educativa y Docencia En Red (IN-RED 2015). Valencia, pp. 1-13.

SANTAFÉ-MOROS, A., GOZÁLVEZ-ZAFRILLA, J.M., NAVARRO-LABOULAIS, J.M., CARDONA-NAVARRETE, S.C., MIRÓ-HERRERO, R., GARCÍA-DÍAZ, J.C. (2014). "Experiencias de aplicación de la simulación empleando software libre y gratuito en la enseñanza de las ingenierías de la rama industrial" en Jornadas de Innovación Educativa y Docencia En Red (IN-RED 2014). Editorial Universitat Politècnica de València, Valencia, pp. 324-342.

STRIJBOS, J., ENGELS, N., STRUYVEN, K., (2015). "Criteria and standards of generic competences at bachelor degree level: A review study". Educ. Res. Rev. 14, 18-32. 